\title{
MÉMOIRE DISCURSIVE ET PÉRÉGRINISMES : VIVIERS D'UNE VARIÉTÉ DE FRANÇAIS IVOIRIEN
}

\section{RABÉ, Charles Liagro ${ }^{1}$}

RÉSUMÉ : La Côte d'Ivoire fait partie des pays francophones où le français, en tant que langue officielle, n'est conjugué à aucune langue nationale. Toutefois, au contact des langues nationales ivoiriennes, le français de référence sinon se métamorphose, du moins se scinde en variétés diverses. Ces dernières se caractérisent par de nombreux facteurs parmi lesquels les emprunts aux langues ivoiriennes, la création de mots hybrides, la création de nombreux synonymes, etc. La variété que nous étudions dans cette réflexion est celle qui se singularise d'abord par la resémantisation de certains termes du français qui deviennent alors polysémiques de sorte que le décryptage des énoncés qui les portent appelle que les coénonciateurs aient en partage une certaine mémoire discursive, ensuite par l'ingestion d'items patrimoniaux interjectifs ouvrant la fenêtre à la glottophagie de la langue française non selon le concept de Jean-louis Calvet mais suivant la conception de Pierre N'da. Au moyen de la pragmatique lexiculturelle et à partir d'énoncés oraux divers et d'extraits de compositions musicales d'artistes ivoiriens, ce travail révèle les procédés linguistiques par lesquels la mémoire discursive et les pérégrinismes, vecteurs a-normatifs, enrichissent la langue française.

MOTS-CLÉS : langue nationale, français, mémoire discursive, pérégrinisme, interjection.

\section{DISCURSIVE MEMORY AND PEREGRINISMS : VIVIERS OF A VARIETY OF IVOIRIAN FRENCH}

\footnotetext{
${ }^{1}$ Enseignant-Chercheur à l'Université Peleforo Gon Coulibaly de Korhogo (Côte d'Ivoire) ; MaîtreAssistant du CAMES (Conseil Africain et Malgache pour l'Enseignement Supérieur) ; Spécialité : Grammaire et Linguistique du français. E-mail : rbecharles@gmail.com
} 
ABSTRACT : Ivory Coast is one of the French-speaking countries where French, as an official language, is not combined with any national language. However, in contact with Ivorian national languages, reference French if not metamorphoses, at least splits into various varieties. The latter are characterized by many factors, including borrowings from Ivorian languages, the creation of hybrid words, the creation of many synonyms, and so on. The variety that we study in this reflection is the one singled out first of all by the resemantization of certain French terms which then become polysemic so that the deciphering of the statements that carry them calls that the co-enunciators share a certain memory discursive, then by the ingestion of interjective patrimonial items opening the window to the glottophagy of the French language not according to the concept of Jean-louis Calvet but according to the conception of Pierre N'da. By means of lexicultural pragmatics and from various oral statements and excerpts of musical compositions by Ivorian artists, this work reveals the linguistic processes by which discursive memory and peregrims, a-normative vectors, enrich the French language.

KEYWORDS : national language, French, discursive memory, peregrinism, interjection.

\section{INTRODUCTION}

Dans la préface du Petit recueil d'ivoirismes de (BOHUI, 2013), le linguiste ivoirien Jérémie Kouadio N'guessan affirme qu'

Après des années de débats, parfois stériles, autour de la reconnaissance des pratiques langagières propres à certains pays d'Afrique francophone, plus personne aujourd'hui ne conteste l'existence de variétés de français, produits d'une acclimatation «chaotique» de cette langue aux conditions écolinguistiques et « écoculturelles » africaines .

La Côte d'Ivoire, de ce point de vue-là, fait partie des pays où ces pratiques diversifiées du français sont attestées de longue date. La variété que nous nous proposons d'étudier dans cette réflexion est celle qui s'alimente de pérégrinismes et scelle une certaine mémoire discursive commune par le recours à des emplois particuliers. Se pose alors la question de la configuration et du fonctionnement desdits emprunts ainsi que des enjeux qui en découlent. Nous appuyant sur la taxinomie 
prescrite par (CHEVALIER, BENVENISTE, ARRIVE, PEYTARD, 1997) relativement aux parties du discours classiques, nous mènerons cette analyse par le recours à la pragmatique lexiculturelle. Galisson note «qu'elle s'adonne prioritairement à l'étude des signes en situation, donc aux rapports qu'ils entretiennent avec les locuteurs et qui les créditent d'un supplément de contenu, précieux révélateur culturel $»^{2}$. Le corpus allie propos oraux divers et extraits de compositions musicales d'artistes ivoiriens. Dans sa structure ternaire, l'article analysera l'emploi du déictique spatial « là », le fonctionnement syntaxique de certains adverbes et deux interjections locales.

\section{I- Le morphème là dans le jeu de la mémoire discursive.}

Avant d'appréhender le morphème là dans son fonctionnement normatif puis dans celui adossé à la mémoire discursive, il convient de définir à gros traits ce qu'évoque la notion de mémoire discursive.

\section{I-1-Approche définitionnelle de la mémoire discursive partagée}

La communication verbale repose en partie sur des savoirs partagés que de nombreuses théories linguistiques intègrent à leur modèle, en particulier, dans le champ du discours. Ces savoirs partagés constituent un ensemble de connaissances valides, pour des interlocuteurs, qui prend le nom de mémoire discursive partagée. Yao Kouamé présente la mémoire discursive «comme une réserve mémorielle, un stock de signifiés dans lequel tout locuteur puise pour asseoir le caractère ternaire de la désignation $»^{3}$, savoir le signifiant, le signifié et le référent. Il rejoint ainsi (CHARAUDEAU, 2002, p. 371). Selon ce dernier, on «parle parfois de mémoire discursive pour rendre compte de l'accroissement progressif des savoirs partagés par les interlocuteurs au cours d'un échange ». Par exemple, le GN « Accord Politique de Ouagadougou (APO) » est un signifiant qui fonctionne de pair avec son signifié et son

\footnotetext{
${ }^{2}$ Robert Galisson, «La pragmatique lexiculturelle pour accéder autrement à une autre culture, par un autre lexique », Mélanges, CRAPEL, n. 25, pp. 47-73.

${ }^{3}$ Yao Kouamé, « Mémoire discursive et structure hypotypotique d'une variété de français ivoirien » in Français et langues nationales en Afrique à l'ère postcoloniale : bilan et perspectives, sous la direction de Jean-Paul Balga et David Abaïkaye, Paris, L’Harmattan, 2017, pp. 91-101.
} 
référent. En raison du caractère notoire du référent de ce syntagme nominal, les ivoiriens et les autres habitants de la Côte d'Ivoire auront immédiatement à l'esprit la discussion directe entre le Président ivoirien Laurent Gbagbo, le chef des Forces Nouvelles Guillaume Soro et le Président Blaise Compaoré laquelle discussion a abouti à la signature, le 4 mars 2007, d'un accord portant sur le processus électoral, le désarmement et la réunification du pays.

Il en va tout autrement du « Cadre Permanent de Concertation (CPC) » créé à l'Université Peleforo Gon Coulibaly de Korhogo pour favoriser le dialogue entre Enseignants et Administration en vue de prévenir les dysfonctionnements de l'institution. Le référent de ce syntagme nominal ne bénéficie pas de la même notoriété que l'APO. Pour cette raison, dans un contexte communautaire plus restreint, il sera difficile à quiconque qui ne détient pas l'information relative au CPC de lui associer un référent précis. Cela consacre l'instabilité référentielle qui fonctionne, ainsi que le dit (BUSCAIL, 2013, p. 122), comme la déixis. Contrairement au premier syntagme nominal qui puise dans la mémoire discursive des ivoiriens, le CPC pourrait paraître comme un déictique, c'est-à-dire comme un signe linguistique dont le sens est fonction des circonstances de l'énonciation. Dans les deux cas cependant, l'appréhension du référent demeure contextuelle. C'est le contexte discursif qui balise la récupération des référents dans un stock ou un paradigme mémoriel propre à la communauté discursive. On voit bien que la mémoire discursive est en lien étroit, non seulement avec les conditions sociales, historiques et cognitives de production des énoncés, mais aussi avec les facteurs «extradiscursifs et prédiscursifs », au sens de (PAVEAU, 2006, p. 252), facteurs qui participent aux productions verbales. Cette brève esquisse définitionnelle autorise à présent l'examen de l'item là.

\section{I-2-Fonctionnement canonique du déictique « là »}

Même si, selon le dictionnaire du $\mathrm{CNRTL}^{4}$, « là » peut fonctionner soit comme un adverbe de lieu, soit comme une particule renforçative d'un démonstratif, soit comme une interjection, on retient surtout qu'en français le morphème « là » est un déictique spatial. À propos du déictique, l'avis de (ORECCHIONI, 2006, p. 42) est qu'il est

${ }^{4}$ CNRTL signifie Centre National de Ressources Textuelles et Lexicales 
sémantiquement stable en raison de l'invariabilité de sa dénotation. Seul son référent varie selon la situation d'énonciation. Le déictique est particulièrement tributaire de son contexte d'apparition dans l'énoncé et se range dans trois catégories. Ce sont le déictique actantiel (da), le déictique spatial (ds) et le déictique temporel (dt) qui sont respectivement en lien avec les composantes même de la déixis connaitre la personne (je et tu), l'espace (ici, là) et le temps (aujourd'hui, maintenant). Le décryptage des déictiques appelle que la situation d'énonciation indique respectivement qui dit « je », l'endroit où il se trouve en disant « je » et le moment où il le dit. En supposant, comme c'est le cas généralement, excepté la communication au téléphone et dans l'obscurité, que l'auditeur voit et entend le locuteur, un enseignant qui se trouve à l'Université Peleforo Gon Coulibaly de Korhogo, le mardi 03 juillet 2018, devant la salle B du bloc $\mathrm{TD} / \mathrm{TP}$, pour indiquer son bureau en vue de fixer un rendez-vous à un groupe d'étudiants peut dire :

(1) Rejoignez-moi là demain soir.

Dans cet exemple, le référent de « là », présent dans l'énonciation, est rendu saillant grâce aux coordonnées contextuelles. Et comme pour s'inscrire dans la logique de (DELPLANQUE, 2012, p. 29) qui affirme que « "Là" désigne un endroit qui, quelle que soit la distance, est distinct mais non séparé du lieu de l'Énonciateur», l'enseignant-locuteur peut joindre « le geste à la parole, surtout lorsqu'il faut identifier un être ou un objet parmi plusieurs »(GREVISSE, 2008, p. 617). Ainsi, il prononcera sans doute cet énoncé avec un index qui pointe vers le bureau qui paraît sous les yeux de ses interlocuteurs. Là est le fonctionnement normatif et référentiel auquel le français ivoirien substitue un fonctionnement mémoriel dans lequel « Là » subit une hypostase, perd son statut de déictique spatial pour revêtir d'autres allures.

\section{I-3- Fonctionnement ivoirisé de l’item « là »}

En français ivoirien, le morphème «là » fonctionne tantôt comme un déterminant syntagmatique, tantôt comme un signe de ponctuation, tantôt comme un item à visée phatique ou explétive, tantôt encore comme une locution prépositive. 


\section{I-3-1-L’item « là » comme un déterminant syntagmatique}

Souventes fois, l'item « là » se comporte comme un déterminant syntagmatique. Soit l'énoncé :

(2) Où est la clé là ?

Cet énoncé pourrait émaner d'un locuteur prêt à monter à bord de sa voiture, mais ne retrouvant pas la clé de contact. S'il pose le problème des niveaux de langue, cet énoncé soulève également la question du statut de l'item « là ». Cet autre exemple pourrait nous éclairer :

(2') Où est la clé de contact de ma voiture ?

En rapport avec le premier exemple, supposons que le «là » soit pris pour un déictique spatial. Dans ce cas, il indiquerait l'endroit où se trouve la clé. Comment dès lors pourrait-on la chercher au moyen de la question posée ? On tomberait dans ce cas sur un non-sens, ce qui rendrait l'interrogation elle-même inutile. Ici, l'item « là » ne peut se manifester que comme un actualisateur, c'est-à-dire comme un déterminant, car il est fortement lié au syntagme nominal « la clé ». Le « là » fournit des précisions référentielles qui lèvent le voile sur la sémantique du syntagme nominal « la clé ».

En produisant l'énoncé de l'exemple 2, le locuteur sait pouvoir compter sur la mémoire discursive de son interlocuteur qui est ainsi chargé, compte tenu de l'énonciation, d'identifier le référent. Ce genre d'énoncés, inondés de «là » où le locuteur sollicite la mémoire discursive de l'allocutaire en vue de débusquer efficacement le référent sont légion dans le français ivoirien. On peut avoir :

(3) Tu as vu ma cravate /mon téléphone là ?

(4) Regarde mon enfant là.

Si l'opération de décodage référentiel devient ardue pour l'auditeur, le sujet locuteur peut recourir aux modificateurs pour le balisage sémantique. On pourrait alors avoir :

(3') Tu as vu ma cravate/ mon téléphone que je viens d'acheter?

(4') Regarde mon enfant dont j'ai dit qu'il est malade.

Les relatives (que je viens d'acheter/dont j'ai dit qu'il est malade) pourraient servir d'adjuvants au co-énonciateur pour réussir le décodage de l'énoncé. 


\section{nonplus}

Quant au niveau de langue relâché, il est l'expression achevée d'une variété de français ivoirien qui consacre ce qu'Anoh appelle « l'économie langagière ${ }^{5}$ ».

Nous abordons à présent la seconde vêture du morphème «là » dans son fonctionnement ivoirisé.

\section{I-3-2-L'item « là » comme un signe de ponctuation}

La ponctuation est devenue un élément essentiel de la langue écrite. (RIEGEL, PELLAT, RIOUL, 2005) la définissent comme « le système des signes graphiques qui contribuent à l'organisation d'un texte écrit et qui apportent des indications prosodiques, marquent des rapports syntaxiques ou véhiculent des informations sémantiques. » Ils ajoutent que ces signes de ponctuation « peuvent correspondre à des phénomènes oraux (pause, intonation) ». C'est dire que les signes de ponctuation indiquent les endroits où, au cours de la lecture d'un texte, il faut éventuellement marquer une pause, la durée de celle-ci et peut-être aussi la nécessité d'infléchir le ton.

Soit la phrase :

(5) Quand je suis arrivé à Korhogo, je suis tombé malade.

Celui qui lit mentalement cette phrase remarquera les deux signes de ponctuation que sont la virgule et le point final. Mais s'il la lit à haute voix pour d'autres personnes, sa voix doit reproduire la signification de la ponctuation insérée dans la phrase. De même si cet énoncé provient d'un locuteur dans une conversation orale, il laissera s'éteindre légèrement le volume de sa voix à la fin du mot « korhogo » en raison de la virgule qui y figure et marquera une pause relativement longue après avoir prononcé le mot «malade » qui est ponctué par le point.

Considérons à présent cet autre énoncé :

(5') Quand je suis arrivé à Korhogo là je suis tombé malade.

C'est un truisme de dire que dans cet énoncé, « là » ne saurait être ni un déictique spatial, ni un actualisateur. Ni un déictique spatial parce que « Korhogo » désigne déjà un lieu géographique, ni un actualisateur parce qu'en tant que substantif toponymique,

\footnotetext{
${ }^{5}$ Joseph Adjé Anoh, « Il a voyagé : une expression problématique dans le parler ivoirien », Revue Ivoirienne des Lettres, Arts et Sciences humaines, 2005, p.27.
} 
« Korhogo » jouit déjà d'une valeur spécifiante. On pourrait donc faire correspondre « là » à la virgule qui figure dans l'énoncé (5). En effet, la production orale de l'énoncé $\left(5^{\prime}\right)$ omettra de marquer une courte pause après le «là ». Le temps de prononciation $\mathrm{du}$ « là » équivaudra sans doute à la courte pause exigée par la présence de la virgule dans l'énoncé (5). Ce fonctionnement de l'item « là » se vérifie dans des énoncés comme :

(6) Quand j'ai fait dormir l'enfant dans la chambre là j'ai failli dormir aussi.

S'il est vrai que la possibilité existe ici d'appréhender «là » comme un actualisateur, il n'en demeure pas moins vrai que cet item fonctionne aussi comme s'il avait remplacé la virgule dans l'énoncé écrit correspondant :

(6') Quand j'ai fait dormir l'enfant dans la chambre, j'ai failli dormir aussi.

De là nous pouvons inférer que dans le français ivoirien, le morphème « là » fonctionne parfois comme un signe de ponctuation. À présent, nous nous intéressons à la troisième vêture de « là » dans le français ivoirien.

\section{I-3-3-Fonctionnement de « là » comme un item à visée phatique, explétive ou comme une locution prépositive}

Proposant une « analyse des termes constitutifs de tout procès linguistique, de toute communication verbale»(BAYLON, FABRE, MIGNOT, 1999), (JAKOBSON, 1963) a élaboré le schéma, devenu traditionnel, de la communication linguistique qui détermine ce que l'on peut faire en se servant de la parole. À «chaque terme constitutif » du procès linguistique est rattachée une fonction. Se dégagent ainsi six fonctions du langage. L'une d'elle, la fonction phatique, « centrée sur le contact entre le destinateur et le destinataire, (...) établit, maintient, interrompt ce contact par des éléments tels que allô, n'est-ce pas, hein, ou, dans la graphie, le point /. / » (ARRIVE, GADET, GALMICHE, 1986). Par exemple, un interlocuteur qui finit le travail à 18h45minutes et à qui le locuteur propose un rendez-vous pour 19h dans un endroit assez éloigné de son lieu de travail, pensant à la probabilité d'accuser du retard pourrait hésiter avant de donner son accord. Il peut alors émettre l'énoncé qui suit :

(7) $19 \mathrm{~h}$ là..., bon, je vais venir. 


\section{nonplus}

Dans cet énoncé, « là » a une fonction qui vise au maintien du contact acoustique entre le locuteur et son interlocuteur, celui-ci se donnant quelque temps de réflexion pour faire droit ou non à la requête de celui-là. On peut également considérer cet énoncé :

(8) Arrête là ; tu me fatigues avec tes histoires de football là.

Cet énoncé nous semble en tous points identiques à celui-ci :

(8') Arrête n'est-ce pas de me fatiguer avec tes histoires de football n'est-ce pas.

Les différentes occurrences de la locution «n'est-ce pas»dans cet énoncé constituent ce que (DURRER, 1999, p. 14) appelle des « ratés conversationnels » et elle s'en explique : "Dans une conversation, l'élaboration d'un message et sa production ont en général lieu simultanément. Les locuteurs ont peu de temps pour planifier leur discours ». Cet avis que nous trouvons compétent se positionne comme un adjuvant à la fonction phatique que Jakobson attribue à cette locution «n'est-ce pas ». Voici ce que nous voulons dire. Étant donné que «les locuteurs ont eu peu de temps pour planifier leur discours ", l'expression manque parfois de fluidité et le plus sûr moyen de maintenir le contact avec l'interlocuteur pour éviter que ses pensées ne vagabondent, c'est de parsemer l'énoncé d'expressions de ce genre. Nul besoin d'en dire davantage pour évoquer la fonction phatique de la locution «n'est-ce pas » dans notre énoncé. Et si, ainsi que nous le pensons, l'énoncé (8') est équivalent à l'énoncé (8), nul besoin non plus d'en dire davantage pour admettre que l'item «là », correspondant pour ainsi dire à la locution «n'est-ce pas » a une visée phatique dans l'énoncé (8). Et que dire du caractère explétif de « là »?

Selon Le grand Robert de la langue française, est qualifié d'explétif tout mot « qui sert à remplir la phrase sans être nécessaire au sens. » Le dictionnaire du CNRTL ${ }^{6}$ ajoute qu'en grammaire, l'explétif est un mot « qui est inutile au sens ou n'est pas exigé par la syntaxe ».

Dans le français ivoirien le morphème « là » fonctionne parfois comme un explétif. C'est le cas dans les questions

(9) Où tu vas /es là ?

\footnotetext{
${ }^{6}$ CNRTL signifie Centre National de Ressources Textuelles et Lexicales
} 
Et dans les réponses

(9') Je vais / suis à l'UPGC là.

Ici, les co-énonciateurs sollicitent leur mémoire discursive partagée pour saisir l'inanité du « là » et ainsi, se comprennent parfaitement.

C'est encore la mémoire discursive qui est mise à contribution lorsqu'il faut entrer dans l'intelligence d'énoncés comme :

(10) Qu'est-ce que tu es là faire ?

(11) Je suis là manger/ travailler.

Ici, la seule analyse qui vaille quant à l'expression à laquelle le français ivoirien a substitué « là », c'est la locution prépositive « en train de » dont Le grand Robert de la langue française dit qu'elle signifie «en cours». Plus simplement, les coénonciateurs discernent qu'il s'agit de

(10') Qu'est-ce que tu es en train de faire?

(11') Je suis en train de manger / travailler.

Outre le déictique là, d'autres items ayant recours à une culture partagée par les locuteurs se meuvent dans le français ivoirien.

\section{II-Examen de quelques expressions dans la dynamique mémorielle}

Cette partie de notre réflexion vise à analyser le fonctionnement de certaines expressions qui confèrent aux énoncés dans lesquels elles apparaissent un sens qui n'est décryptable que dans la dynamique mémorielle. Il s'agit de « mal même » et «je vais / ça va tuer ».

\section{II-1- L'expression « mal même » dans le moule antiphrastique}

L'expression «mal même » est de toute évidence la somme de deux adverbes : mal et même. Chacun sait que l'adverbe mal s'emploie en général pour évoquer ce qui choque le goût et les convenances, ce qui est fait d'une manière défectueuse et imparfaite, d'une façon éloignée de la normale, ainsi que le montrent respectivement les exemples suivants :

(12) Individu mal élevé.

(12’) Voiture mal réparée. 
(12’') Femme mal bâtie.

Avec ces différents sens, l'adverbe mal se pose comme étant le contraire de l'adverbe bien, ce qui est bien évoquant dès lors ce qui convient, ce qui est fait d'une manière satisfaisante, parfaite ou d'une manière qui plaît.

Quant à l'adverbe même, il s'emploie souventes fois avec une valeur de renchérissement et de renforcement. C'est le cas dans

(13) Son savoir-faire étonnait même les personnes expérimentées.

Cela dit, on devrait s'attendre du point de vue de la logique conversationnelle ${ }^{7}$, à ce que la conjugaison de mal et même en «mal même » évoque, avec force, ce qui est contraire à ce qui est bien. Or, qu'en est-il du sens de cette expression en français ivoirien?

Supposons que dans le cadre d'une présélection, un sélectionneur n'ayant jamais vu jouer les personnes qui lui sont proposées demande à l'une d'entre elles :

(14) Sais-tu jouer au football ? Réponse du joueur :

(14') Je joue mal même.

Faut-il comprendre que ce joueur avoue être un très mauvais footballeur ? Loin s'en faut ! Ah, si ; pour le locuteur non initié au français ivoirien, qui ne partage donc pas le stock mémoriel des ivoiriens. Et ce locuteur se méprendrait sur le sens réel de l'énoncé. En effet, le locuteur ivoirien ou vivant en Côte d'Ivoire depuis des lustres comprend que ce joueur, par ce qu'il dit dans l'énoncé de l'exemple (14'), veut dire qu'il joue très bien au football. Le passage du dit au vouloir dire s'orchestre dans la dynamique de la mémoire discursive partagée. Du coup l'énoncé (14'), en raison de l'expression mal même qui emporte le sens contraire à sa signification normée, se saisit comme une antiphrase. Autre expression, autre signification, pourrait-on dire pour ce qui suit.

\section{II-2- L'expression « je vais / ça va tuer » en posture elliptique}

Tuer, chacun le sait, c'est ôter la vie. Ainsi, par exemple, dans l'énoncé

\footnotetext{
${ }^{7}$ Charles Rabé Liagro, «La problématique du langage hypocoristique dans La ronde des jours de Bernard Dadié », in Cahiers du Grems, Revue annuelle du Groupe Recherches en Morphosyntaxe et Sémantique, N02, décembre 2017, p. 152-153.
} 
(15) Je vais tuer deux moutons,

le COD «deux moutons », en tant que complément caractérisant est une simple détermination qui véhicule une information dénotative. C'est lorsqu'il est employé intransitivement que tuer appelle à interprétation car on peut procéder à divers complètements. Je vais tuer... (un animal / le temps). Bien sûr que le sens de l'énoncé variera selon le complément ajouté. Il faut par ailleurs tenir compte de la situation d'énonciation. L'illustration qui suit nous éclairera sur ce point. À l'occasion de l'une des émissions humoristiques organisées par la $\mathrm{RTI}^{8}$ à l'orée de chaque nouvel an, un humoriste ivoirien lance devant le public :

(16) Je vais tuer.

En entendant ces paroles, l'auditoire que de s'effaroucher, s'excite plutôt de joie. C'est dire que l'énoncé n'a rien ni d'effrayant, ni d'odieux. Au contraire, il est plaisant. L'artiste promet ainsi de procurer un grand plaisir par sa prestation. L'expression « je vais tuer » semble donc l'ellipse de « je vais vous tuer de plaisir », ou «ça va tuer» c'est-à-dire «ce que je vais faire va vous tuer de plaisir». Plus simplement, la prestation va susciter une telle joie que les spectateurs vont mourir de rire. Une autre analyse qui permet de tyranniser la compréhension ci-devant évoquée concerne un énoncé qui voisine avec le précédent. Il s'agit de cet énoncé extrait du titre « Le vieux est trop fort » du groupe musical ivoirien LES PRO DU ZOUGLOU :

(16') Quand il a dit ça, son fan club à côté, eux ils veulent mourir'.

Restituons à cet énoncé son contexte. Cette chanson relate l'histoire d'un vieil homme qui a remporté une victoire difficile. Le concours consistait à révéler le nom des boissons alcoolisées auxquelles les prétendants au prix goutent, les yeux fermés. Tous les participants ayant échoué, cet homme d'âge a décidé de prendre part à la compétition et a réussi à indiquer le nom de toutes les boissons qui lui ont été servies.

Les organisateurs, malhonnêtes, voulant coûte que coûte le faire échouer ont imaginé un stratagème. Comme dernière, épreuve, ils lui ont servi, non pas de la boisson alcoolisée comme le voulaient les règles du jeu, mais l'eau ayant servi à laver l'intérieur d'une vieille chaussure. À la stupéfaction générale, « le vieux », révèle la

\footnotetext{
${ }^{8}$ RTI : Radiodiffusion Télévision Ivoirienne

${ }^{9}$ LES PRO DU ZOUGLOU, titre : « Le vieux est trop fort » extrait de l'album « notre voie », 2018.
} 
nature et l'origine de la boisson servie mais bien plus, la marque de la chaussure ainsi que l'état déplorable de cette dernière. Ivres de bonheur, les supporters du vieil homme ne pouvaient plus contenir leurs sentiments. C'est alors qu'ils déversent à larges effluves l'immense et intarissable joie qu'ils avaient sur le reste de la foule.

C'est donc pour rendre compte de ce bonheur qui jaillissait avec des bouillonnements joyeux des supporters du vieil homme que l'énoncé (16') est produit. Du coup, on saisit sans peine que les membres du « fan club» «veulent mourir », pas au sens de s'ôter la vie, mais de savourer la victoire du vieil homme en donnant libre cours à leur joie. Cette analyse trouve également son fondement dans le clip vidéo qui accompagne l'énoncé « ils veulent mourir », lequel clip présente les membres du «fan club» dansant et gambadant de joie. En guise d'explication de l'expression « ils veulent mourir », ce clip semble dire : regardez comment les membres du fan club du «vieux» sont en train de «mourir» de joie. Et c'est parce que l'expression « ils veulent mourir » est amputée du syntagme prépositionnel « de joie» que nous l'appréhendons sous le prisme elliptique. Il est évident qu'une telle compréhension de l'expression puise à la fois dans la situation d'énonciation et dans la mémoire discursive.

En plus du morphème « là », des expressions «mal même », «je vais tuer » et de son dérivé « ils veulent mourir » dans le jeu de la mémoire discursive en parler ivoirien, des items patrimoniaux alimentent le français ivoirien.

\section{III- Interjections locales et français : « pour quel camembert ! ?»}

Dans cette partie, nous nous proposons d'examiner le mécanisme par lequel les interjections territoriales « 000 » et « dêh » tropicalisent le français de référence. Il convient pour cela de faire remarquer hic et nunc que même si le « ooo » patrimonial a une image sonore proche de celle du «oh» de référence, il s'en distingue par l'orthographe - c'est trivial - par les traits intonationnels et par le fonctionnement syntaxique. C'est qu'au niveau syntaxique, le «ooo» patrimonial ne se range qu'en fin de phrase contrairement au «oh » référentiel qui se prépose presque toujours au reste de la phrase et qui, quelque rares fois se loge partout ailleurs dans la structure phrastique. 


\section{III-1- Fonctionnement de l'interjection « 000 »}

Dans une réflexion que nous avons menée sur les interjections en 2016, nous avions déjà évoqué en partie le fonctionnement de « 000 ». Voici ce que nous en avons dit : «Mahouooo se compose du désignateur rigide Mahou qui signifie « je suis mort » et du suffixe ooo qui est un cri chargé dans sa réalisation phonétique de porter loin la voix en vue d'appeler au secours. ${ }^{10} »$ Nous avons qualifié cette interjection de «cri de détresse » susceptible de « faire vibrer la fibre maternelle africaine. » Toutefois, il convient d'admettre que pour le besoin de l'analyse, nous avons dû dessouder le désignateur rigide «mahou » et son suffixe « 000 ». Ce n'était donc là qu'un emploi spécifique de cet item adossé à une situation d'énonciation bien déterminée. Pourtant, il est des moments et c'est assez fréquent, où l'interjection « ooo » assume seule son aventure. C'est de cela que parle la présente analyse.

\section{III-1-1 « 000 » comme demande de compassion et d'attention}

Supposons qu'un locuteur, après avoir adressé une salutation à son interlocuteur, lui demande :

(10) Comment ça va ?

La réponse pourrait être

(11') Ça va bien ooo.

Le locuteur qui, suite à cette réponse de l'énoncé (11'), met fin à l'échange ou aborde aussitôt un autre sujet fournit implicitement la preuve qu'il ne partage pas le même paradigme mémoriel que son interlocuteur. Ce locuteur pourrait ne pas être un ivoirien ou un habitant de la Côte d'Ivoire.

A contrario, le locuteur ivoirien ou vivant en Côte d'ivoire sera interpellé par l'interjection « 000 ». Mise à contribution, sa mémoire discursive lui rappellera que ce « ooo» descendant, on peut en dessiner la courbe ainsi $\downarrow$, mérite décryptage. L'énoncé se fut terminé par l'adverbe « bien » qu'on n'eût pas besoin d'épiloguer sur son sens, car on eût compris que l'émetteur du « Ça va bien » se porte tout simplement bien.

${ }^{10}$ Charles Liagro Rabé, «Les interjections, des convoyeurs culturels dans La carte d'identité de JeanMarie Adiaffi », in Lettres d'Ivoire, Université Alassane Ouattara, N 023, septembre 2016, p. 102. 
Dans le contexte du français ivoirien, la présence du « 000 » descendant dans l'énoncé (11') peut donner lieu à plus d'une interprétation. «Ça va bien ooo » peut donc signifier :

- Je ne me porte pas très bien.

- Je ne me porte pas à merveille, mais mon état de santé n'est pas alarmant.

- Même si ce n'est pas la grande santé, je me sens mieux qu'il y a deux jours.

- Pour ma santé, que dire si ce n'est de vouloir ce qu'on ne peut empêcher.

- Je me contente de ce que Dieu m'accorde comme état de santé.

- Il faut faire contre mauvais sort bon cœur.

- Je fais tant bien que mal face à des soucis financiers / familiaux.

Ces possibles interprétations ont ceci de commun que le « ooo » nuance le contenu sémantique de l'adverbe « bien » et en prend même le contre-pied. Grâce à sa mémoire discursive, le locuteur ivoirien ou vivant en Côte d'Ivoire ayant perçu ce champ sémantique, s'enquerra sans doute :

(10) Qu'est ce qui ne va pas?

Cette seconde question qui est l'expression d'une marque d'intérêt donnera à l'interlocuteur l'occasion de préciser son souci. Cela peut être :

- Je me remets progressivement de mon mal mais c'est si lent...

- Mon traitement est efficace mais j'ai besoin d'une petite somme d'argent pour acheter le reste des médicaments.

- Je ne plains pas ma santé mais je rencontre quelques problèmes financiers / familiaux...

On voit bien à travers ces différents possibles, que l'emploi du « ooo » peut évoquer tantôt l'état de santé de l'émetteur de l'énoncé, tantôt sa situation financière ou familiale délétère, tantôt encore une sollicitation financière. Dans tous ces possibles, le « ooo » descendant s'apparente à un appel que lance le locuteur afin de solliciter de son interlocuteur la compassion, l'attention et l'attendrissement.

Bien sûr que le champ d'interprétations s'amincit à mesure que la situation d'énonciation se circonscrit. En effet, si le «Comment ça va ?» de l'énoncé (11) est 
adressé à un étudiant qui sort d'une salle de composition, le « Ça va bien ooo » de ce dernier sera nécessairement et absolument en lien avec son examen.

\section{III-1-2- « 000 » comme expression d'affection}

Nous nous intéressons à présent à un autre emploi de l'interjection « 000 ». Considérons à cet effet l'énoncé suivant où une mère appelle sa fille Ruth.

(10) Ruth ooo, viens ooo. (Lire/ryto vjêo/ avec une courbe mélodique descendante $\downarrow$

L'énoncé (13), avec son ton descendant où le «ooo » se fond dans le nom « ruth » et dans le verbe «viens ", est l'expression achevée dans le français ivoirien de l'affection que la mère locutrice voue à sa fille. L'interjection « ooo », dans ce cas, est donc un accent d'affection et d'amour.

\section{III-1-3- « 000 » comme indice distanciel}

Avec l'énoncé qui suit, la mère appelle encore Ruth mais cette fois avec un trait intonationnel différent :

(10) Ruth ooo !viens ooo ! (Lire / ryt o vjê o/ avec une courbe mélodique montante $\uparrow$

Le ton ascendant de l'énoncé (14) prouve que l'appel dirigé vers Ruth est effectué avec un puissant volume de la voix. Ici, le « ooo » sert à porter loin la voix de la mère locutrice, preuve que la réceptrice se trouve à une assez longue distance de l'endroit à partir duquel l'appel est émis. Preuve également qu'à l'instar de la plupart des langues africaines qui sont essentiellement tonales, le français ivoirien est imprégné d'oralité africaine.

« ooo » n'étant que le premier brin de la paire d'interjections territoriales à l'étude, nous abordons à présent le second.

\section{III-2- Fonctionnement de l'interjection « dêh »}

Contrairement à l'interjection locale « ooo» qui voisine quelques fois phonétiquement avec le «oh » du français de référence, le «dêh » lui, n’a aucune 
commune mesure avec le lexique français. Pour cette raison, nous trouvons opportun d'en présenter l'origine.

\section{III-2-1- Présentation de l'interjection « dêh »}

Issu du Jula, langue commerciale ivoirienne parlée à travers tout le pays, « dêh [est un] exclamatif »(BOHUI, 2013, p. 27), d'intensité qui se place en fin de phrase. Voici par exemple un énoncé Jula :

(10) a ka di dêh !

et son correspondant français :

(15') Ce repas est très bon! Qu'est-ce que ce repas est bon !

Ici, le « dêh » met en relief la qualité du repas. Un autre énoncé Jula :

(10) I fasara dêh !

(16') Tu as vraiment maigri !

Ici encore, l'exclamatif d'intensité « dêh » évoque un amaigrissement accentué. Cette brève présentation autorise, pensons-nous, à appréhender «dêh » dans une combinatoire phrastique française.

\section{III-2-2- « Dêh » comme expression d'une valeur ajoutée sémantique}

Lorsque l'item Jula « dêh » se fait de la place dans le français ivoirien, il conserve fondamentalement son sens accentuel, même si dans certains contextes d'énonciation, l'accentuation qu'il marque se fait plus insidieuse. Si à un emprunteur qui promet de rembourser à une certaine date, le prêteur dit :

(10) Il faut respecter la date dêh !

le «dêh » insiste il est vrai, sur l'importance du remboursement à la date prévue, mais il revêt également des allures de mise en garde comme si cet emprunteur avait déjà manqué à sa parole ou comme s'il manquait de conviction en faisant la promesse. Une analyse similaire vaut pour le vers de l'artiste ivoirien Dezy Champion :

(11) Trop jaloux tu deviens sorcier dêh ${ }^{11}$ !

${ }^{11}$ Désiré Claver Guinon surnommé Dezy Champion, titre de la chanson, « Jalousie » extrait de l'album «Orphelin », 2012. 
Chacun sait qu'amputé de l'exclamatif patrimonial « dêh », ce vers devient un énoncé purement informatif. Le « dêh », comme dans l'exemple (17), charrie donc une valeur ajoutée sémantique qui ici, imprime à l'énoncé un cachet d'avertissement lié à la dangerosité de la sorcellerie. Ce que nous appelons valeur ajoutée sémantique induite par «dêh » est en fait une glottophagie de la langue française. Certes, cette expression de glottophagie a été employée et vulgarisée par le linguiste français (CALVET, 1974) dans Linguistique et colonialisme pour décrire le viol des langues africaines par celles des colonisateurs européens. Toutefois, selon (N'DA, 2003, P. 124), on l'emploie aussi pour désigner, en retour, le travail effectué par les écrivains africains sur les langues d'emprunt pour les décoloniser et les re-territorialiser en les indigénisant.

\section{CONCLUSION}

La variété de français ivoirien que nous venons de décrire s'alimente et s'enrichit de facteurs patrimoniaux qui tendent à gouverner son fonctionnement. Pour cette raison, sa configuration syntaxico-lexicale se veut rétive à la syntaxe normative du français de référence. L'enjeu majeur de cette variété demeure le décryptage des énoncés par les co-énonciateurs qui doivent pour cela recourir à une mémoire discursive qui se veut un paradigme de données dont ils doivent partager la connaissance car de cela dépend la réussite communicationnelle. Finalement, tout en se positionnant comme un vecteur d'enrichissement du français normatif, le français ivoirien ne manque pas de révéler son caractère abscons pour le locuteur étranger. Quitte à ce dernier de visiter ce terreau fertile de richesses linguistiques où le normatif est constamment bousculé pour le plus grand bonheur des usagers de la langue française. 


\section{REFERÊNCIAS BIBLIOGRÁFICAS}

ANOH, Adjé Joseph. «Il a voyagé : une expression problématique dans le parler ivoirien ». In : Revue Ivoirienne des Lettres, Arts et Sciences humaines, 2005.

ARRIVE, Michel. Grammaire du français contemporain. Paris : Larousse-Bordas, 1997.

ARRIVE, Michel; GADET Françoise; GALMICHE Michel. La grammaire d'aujourd'hui : guide alphabétique de linguistique française. Paris : Flammarion, 1986.

BAYLON, Christian ; FABRE Paul ; MIGNOT Xavier. Initiation à la linguistique. Paris : Nathan, 1999.

BOHUI, Djédjé Hilaire. Petit recueil d'ivoirismes. Paris : Editions Publibook, 2013.

BUSCAIL, Laurie. «Etudes comparatives des pronoms démonstratifs neutres anglais et français à l'oral : référence indexicale, structure du discours et formalisation en grammaire notionnelle dépendancielle ». In : Linguistics Université Toulouse le Mirail-Toulouse II, 2013.

CALVET, Jean-Louis. Linguistique et colonialisme. Petit traité de glossophagie. Paris : Payot, 1974.

CHARAUDEAU, Patrick. Dictionnaire d'analyse du discours. Paris : Seuil, 2002.

DELPLANQUE, Alain. Forme et Malléabilité : topologie des opérations énonciatives. Université de Ouagadougou, Université de Tours, https:// hal.archives-ouvertes.fr/hal_00745067, 24 octobre 2012.

DURRER, Sylvie. Le dialogue dans le roman. Paris : Nathan, 1999.

GALISSON, Robert. «La pragmatique lexiculturelle pour accéder autrement à une autre culture, par un autre lexique ». In : Mélanges, CRAPEL, n. 25, pp. 47-73.

GREVISSE, Maurice et GOOSSE André. Le bon usage. 14 édition. Bruxelles : De Boek, 2008.

GUINON, Désiré Claver - surnommé Dezy Champion, titre de la chanson, « Jalousie » extrait de l'album « Orphelin », 2012.

JAKOBSON, Roman. Essais de linguistique générale. Paris : Editions de Minuit, 1963. 
KERBRAT-ORECCHIONI, Cathérine, L'Enonciation. Paris : Armand Colin, 2006.

KOUAME, Yao. «Mémoire discursive et structure hypotypotique d'une variété de français ivoirien ». In : Français et langues nationales en Afrique à l'ère postcoloniale : bilan et perspectives, sous la direction de Jean-Paul Balga et David Abaïkaye. Paris : L’Harmattan, 2017, pp. 91-101.

LES PRO DU ZOUGLOU, titre : «Le vieux est trop fort » extrait de l'album « notre voie », 2018.

N'DA, Pierre. L'écriture romanesque de Maurice Bandama ou la quête d'une esthétique africaine moderne. Paris : 1'Harmattan, 2003.

N'GUESSAN, Kouadio Jérémie, dans Préface du Petit recueil d'ivoirismes de BOHUI Djédjé. Paris : Editions Publibook, 2013.

PAVEAU, Marie-Anne. Les prédiscours : Sens, mémoire, cognition. Paris : Presses Sorbonne Nouvelle, 2006.

RABE, Liagro Charles. «Les interjections, des convoyeurs culturels dans La carte d'identité de Jean-Marie Adiaffi ». In : Lettres d'Ivoire, Université Alassane Ouattara, N. 023, septembre 2016, p. 102.

RABE, Liagro Charles. «La problématique du langage hypocoristique dans La ronde des jours de Bernard Dadié ». In : Cahiers du Grems, Revue annuelle du Groupe Recherches en Morphosyntaxe et Sémantique, N. 02, décembre 2017, p.152-153. RIEGEL, Martin ; PELLAT, Jean-Christoph ; RIOUL, René. Grammaire méthodique du français. Paris : Presses Universitaires de France, 2005. 